\title{
Apsidal motion in southern eccentric eclipsing binaries: V539 Ara, GG Lup, V526 Sgr and AO Vel ${ }^{\star}$
}

\author{
M. Wolf ${ }^{1}$ and M. Zejda ${ }^{2}$ \\ 1 Astronomical Institute, Charles University Prague, 18000 Praha 8, V Holešovičkách 2, Czech Republic \\ e-mail:wolf@cesnet.cz \\ 2 Nicholas Copernicus Observatory and Planetarium, 61600 Brno, Czech Republic
}

Received 20 August 2004 / Accepted 20 February 2005

\begin{abstract}
Several new times of minimum light have been observed photoelectricaly for the early-type double-lined eccentric eclipsing binaries V539 Ara $(P=3.2, e=0.05)$, GG Lup $(1.9,0.15)$, V526 Sgr $(1.9,0.22)$ and AO Vel $(1.6,0.07)$. The $\mathrm{O}-\mathrm{C}$ diagrams are analysed using all reliable timings found in the literature and improved values for the elements of the apsidal motion are computed. We find more precise, relatively short periods of apsidal motion of about 162, 102, 155 and 54 years for V539 Ara, GG Lup, V526 Sgr and AO Vel, respectively. The corresponding internal structure constants, $\log k_{2}$, are then found to be $-2.33,-2.16,-2.36$ and -2.24 under the assumption that the component stars rotate pseudosynchronously. The relativistic effects are negligible, being about 6-7\% of the total apsidal motion rate in all systems. Using the light-time effect solution, for V539 Ara we have found a third component orbiting with a period of about 42 years, for AO Vel we confirmed the third body with an orbital period of 33 years.
\end{abstract}

Key words. stars: binaries: eclipsing - stars: individual: V539 Ara - stars: individual: GG Lup - stars: individual: V526 Sgr stars: individual: AO Vel - stars: fundamental parameters

\section{Introduction}

Eccentric eclipsing binaries with apsidal motion are useful sources of knowledge about the internal structure of stars. The motion of the apsis of a binary star is a direct consequence of the finite size of its components. This effect has been well known for decades (Cowling 1938; Sterne 1939). The rate of motion of the apsis is dependent on the internal structure of each component. The study of the apsidal motion thus provides an important observational test of the theoretical models of stellar structure and evolution.

We are interested in an analysis of the light-time effect of multiple stellar systems. The combination of apsidal motion with a light-time effect in such multiple systems serves as an excellent laboratory of celestial mechanics to study a wide variety of processes in stellar astrophysics.

In this paper, we report new results from our project to monitor eclipsing binaries with eccentric orbits (see f.e. Wolf et al. 1999; Wolf 2000). Here we analyse the observational data and rates of apsidal motion for four well-known eclipsing systems, all relatively bright southern-hemisphere objects, known with eccentric orbit and that exhibit apsidal motion. With the exception of AO Vel, spectroscopic observations have been published for all these binary systems.

* Based on observations secured at the South Africa Astronomical Observatory, Sutherland, South Africa.
Our previous photometric study of apsidal motion in southern eccentric eclipsing binaries was published by Wolf (2000). Current O-C diagrams for GG Lup, V526 Sgr and AO Vel were given in Kreiner et al. (2001).

\section{Observations}

The aim of our photometric measurements was to secure several new, well-covered primary and secondary minima for all variables. Our new photoelectric $U B V$ observations were obtained with modular photometer utilizing a Hamamatsu EA1516 photomultiplier on the 0.5-m telescope at the Sutherland site of the South Africa Astronomical Observatory (SAAO) during two weeks in April/May 2004. Each observation of an eclipsing binary was accompanied by observation of a local comparison star (see Table 1). The photoelectric measurements were done in the $U B V$ filters of the Johnson's photometric system with a $10 \mathrm{~s}$ integration time. All observations were carefully reduced to the Cousins E-region standard system (Menzies et al. 1989) and corrected for differential extinction using the reduction program HEC 22 rel. 14 (Harmanec \& Horn 1998). The standard errors of these measurements were about $0.008,0.006$ and $0.005 \mathrm{mag}$ in $U, B$ and $V$ filters, respectively.

The new times of primary and secondary minima and their errors were determined using a least squares fit of the data and 
Table 1. Selected local comparison stars and their colour indexes.

\begin{tabular}{cccccl}
\hline \hline Variable & $\begin{array}{c}\text { Comparison } \\
\text { HD }\end{array}$ & $\begin{array}{c}V \\
{[\mathrm{mag}]}\end{array}$ & $\begin{array}{c}B-V \\
{[\mathrm{mag}]}\end{array}$ & $\begin{array}{c}U-B \\
{[\mathrm{mag}]}\end{array}$ & Remark \\
\hline V539 Ara & 161917 & 6.086 & 0.014 & -0.042 & this paper \\
GG Lup & 136334 & 6.209 & 0.068 & 0.073 & used by Andersen et al. (1993) \\
V526 Sgr & 178110 & 8.745 & -0.006 & -0.266 & used by Lacy (1993) \\
AO Vel & 68657 & 5.821 & -0.152 & -0.627 & used by Grønbech (1985) \\
\hline
\end{tabular}

Table 2. New times of minimum light.

\begin{tabular}{clllc}
\hline \hline System & $\begin{array}{l}\text { JD Hel.- } \\
\text { 2400 000 }\end{array}$ & $\begin{array}{l}\text { Error } \\
\text { [day] }\end{array}$ & Epoch & Source \\
\hline V539 Ara & 48572.799 & 0.005 & 1109.5 & Hipparcos \\
& 48747.100 & 0.005 & 1164.5 & Hipparcos \\
& 48753.443 & 0.005 & 1166.5 & Hipparcos \\
& 53120.4091 & 0.0001 & 2544.5 & SAAO \\
& 53128.440 & 0.002 & 2547.0 & SAAO \\
GG Lup & 48170.351 & 0.005 & 1099.5 & Hipparcos \\
& 53123.5227 & 0.0001 & 3777.5 & SAAO \\
& 53124.6105 & 0.0001 & 3778.0 & SAAO \\
& & & & \\
V526 Sgr & 51342.367 & 0.005 & 1877.0 & NSVS \\
& 51963.349 & 0.002 & 2200.5 & ASAS-3 \\
& 52678.256 & 0.002 & 2573.0 & ASAS-3 \\
& 52829.882 & 0.002 & 2652.0 & ASAS-3 \\
& 53121.6334 & 0.0004 & 2804.0 & SAAO \\
& 53123.5521 & 0.0001 & 2805.0 & SAAO \\
& 53124.6006 & 0.0001 & 2805.5 & SAAO \\
& & & & \\
AO Vel & 51869.382 & 0.005 & 4307.5 & ASAS-3 \\
& 51870.234 & 0.005 & 4308.0 & ASAS-3 \\
& 52405.811 & 0.010 & 4646.0 & ASAS-3 \\
& 52406.563 & 0.005 & 4646.5 & ASAS-3 \\
& 52885.944 & 0.005 & 4949.0 & ASAS-3 \\
& 52886.698 & 0.005 & 4949.5 & ASAS-3 \\
& 53122.8068 & 0.0003 & 5098.5 & SAAO \\
\hline & & & & \\
\hline
\end{tabular}

by the bisecting chord method. Only the bottom parts of the eclipses were used. The given errors represent the third order polynomial fitting mean error for each light curve. In the case of SAAO measurements the mean value and error from the individual filter bands are given. The new times of minima are included in Table 2. In this table, the epochs are calculated from the light elements given in the text, the other columns being self-explanatory.

\section{Apsidal motion analysis}

The apsidal motion in all system was studied by an $\mathrm{O}-\mathrm{C}$ diagram analysis. For an accurate calculation of the apsidal motion rate the method described by Giménez \& García-Pelayo (1983), with equations revised by Giménez \& Bastero (1995), was routinely used. Our relation for the prediction of the times of minimum caused by the apsidal motion is also given in Wolf \& Šarounová (1995).
We have collected all reliable times of minimum light available in the literature and current databases. The ASAS-3 (Pojmanski 2002), Hipparcos (Perryman et al. 1997) and Northern Sky Variability Survey (NSVS, Wozniak et al. 2004) databases have been used to find new times of minimum light. The NSVS observations were collected by the ROTSE-1 experiment using a robotic system of four unfiltered telephoto lenses equipped with CCD cameras. All photoelectric and CCD times of minima were used with a weight of 10 in our computation. Earlier, less accurate photographic or visual measurements were assigned weights of 3 or 1 . We tested the stability of the results with respect to our - somewhat arbitrarily chosen - weighting scheme. It turned out that the results for the well-covered phase curves of all binaries are insensitive to the weighting scheme used.

\subsection{V539 Ara}

The detached double-lined eclipsing binary V539 Ara (also HD 161783, HR 6622A, HIP 87314, CD-537423, CPD-538 899, BV 420, FL 2271; $\alpha_{2000}=17^{\mathrm{h}} 50^{\mathrm{m}} 28^{\mathrm{s}}, \delta_{2000}=$ $\left.-53^{\circ} 36^{\prime} 45^{\prime \prime}, V_{\max }=5^{\mathrm{m}} .71 ; \mathrm{Sp} . \mathrm{B} 3 \mathrm{~V}+\mathrm{B} 4 \mathrm{~V}\right)$ is a frequently studied early-type binary with a slightly eccentric orbit $(e=$ 0.055 ) and an orbital period of about 3.17 days. The double-line spectrum was discovered by Neubauer (1930a), eclipses were later detected by Strohmeier (1964). See also history of work on this binary in Clausen (1996). The secondary component belongs to the slowly pulsating B stars with periods of $1.36,1.78$ and possibly 1.08 days. V539 Ara has a close fainter visual companion HR 6622B $\left(m_{V}=9\right.$.3) which may be physically connected to the eclipsing pair. A circular diaphragm of $35^{\prime \prime}$ was used in our photometric measurements, so this visual companion was included. Clausen (1996) derived the apsidal motion period to be $U=150 \pm 15$ years with a remark that it is not possible to describe all available times of minima through normal apsidal motion.

All photoelectric times of minimum light given in Clausen (1996, his Table 2) were incorporated in our calculation. The epochs in Table 2 were computed according to the ephemeris given in that paper:

Pri. Min. = HJD 2445 056.777 + 3.169112 $\cdot$ E.

Three new times were derived using the Hipparcos photometry (Perryman et al. 1997). A total of 34 photoelectric times of minimum light were used in our analysis, with 16 secondary eclipses among them. The orbital inclination was adopted to be $i=85^{\circ} .2$ based on the photometric analysis (Clausen 1996). As was generally found, the apsidal motion solution is insensitive 
Table 3. Apsidal motion elements of V539 Ara, GG Lup, V526 Sgr and AO Vel.

\begin{tabular}{|c|c|c|c|c|c|}
\hline Element & Unit & V539 Ara & GG Lup & V526 Sgr & $\mathrm{AO} \mathrm{Vel}$ \\
\hline$T_{0}$ & HJD & $2445056.7459(7)$ & 2446136.7448 (1) & $2447739.6517(2)$ & $2445043.6700(3)$ \\
\hline$P_{\mathrm{s}}$ & days & 3.1690854 (12) & $1.8495996(15)$ & $1.91941177(8)$ & $1.5846212(7)$ \\
\hline$P_{\mathrm{a}}$ & days & $3.1692552(12)$ & $1.8496919(15)$ & $1.91947680(8)$ & $1.5847476(7)$ \\
\hline$e$ & & $0.0548(15)$ & $0.1546(10)$ & $0.2204(4)$ & $0.0735(6)$ \\
\hline$\dot{\omega}$ & deg cycle $^{-1}$ & $0.0193(10)$ & $0.0180(5)$ & $0.01219(3)$ & $0.0287(3)$ \\
\hline$\dot{\omega}$ & $\operatorname{deg} \mathrm{yr}^{-1}$ & $2.22(0.1)$ & $3.54(10)$ & $2.320(6)$ & $6.61(10)$ \\
\hline$\omega_{0}$ & deg & $118.9(1.2)$ & $86.12(0.15)$ & $254.9(0.1)$ & $97.1(0.1)$ \\
\hline$U$ & years & $161.8(8.1)$ & $101.6(1.0)$ & $155.2(0.2)$ & $54.4(0.5)$ \\
\hline
\end{tabular}

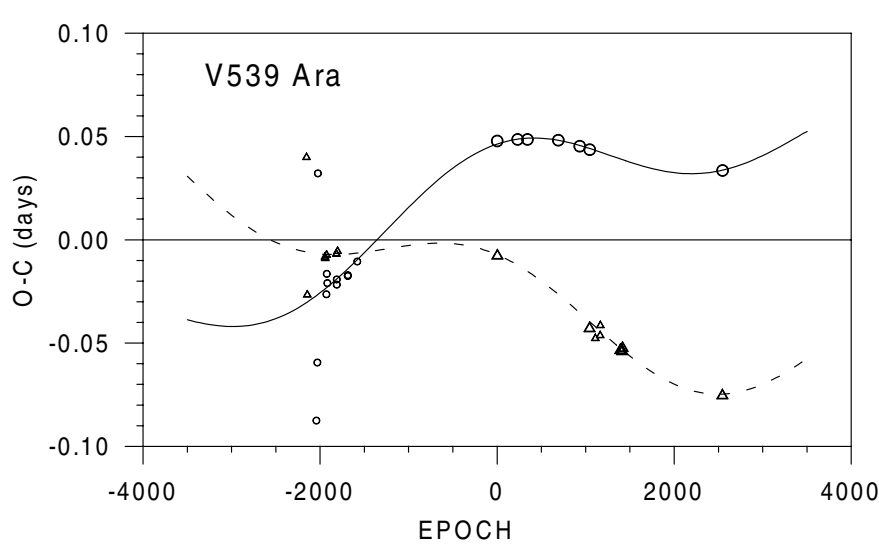

Fig. 1. The O-C diagram for the times of minimum of V539 Ara. The continuous and dashed curves represent predictions for the primary and secondary eclipses. The individual primary and secondary minima are denoted by circles and triangles. Larger symbols correspond to the photoelectric or CCD measurements which were given higher weights in the calculations.

to relatively large changes in $i$, but strongly depends on the orbital eccentricity. Analysing the preliminary O-C diagram, we solved the apsidal motion and the light-time effect (LITE) simultaneously. The deviation of the observed values $(\mathrm{O}-\mathrm{C})_{\mathrm{obs}}$ from the linear ephemeris is given by a superposition of the apsidal motion of the eccentric binary system and by the LITE caused by a presence of a third body:

$(\mathrm{O}-\mathrm{C})_{\mathrm{obs}}=(\mathrm{O}-\mathrm{C})_{\mathrm{aps}}+(\mathrm{O}-\mathrm{C})_{\mathrm{LITE}}$.

The computed apsidal motion parameters and their internal errors of the least squares fit (in brackets) are given in Table 3. In this table $P_{\mathrm{s}}$ denotes the sidereal period, $P_{\mathrm{a}}$ the anomalistic period, $e$ represents the eccentricity and $\dot{\omega}$ is the rate of periastron advance (in degrees per cycle or in degrees per year). The zero epoch is given by $T_{0}$ and the corresponding position of the periastron is represented by $\omega_{0}$. The orbital eccentricity was taken as a free parameter in our calculations. The resulting values of $e$ have smaller intrinsic errors compare to those determined independently from the light curve analysis. This procedure gives us a better result for this important element. The $\mathrm{O}-\mathrm{C}$ residuals for all times of minimum with respect to the linear part of the apsidal motion equation are shown in Fig. 1. The non-linear predictions, corresponding to the fitted parameters, are plotted as continuous and dashed curves for primary and secondary eclipses, respectively.

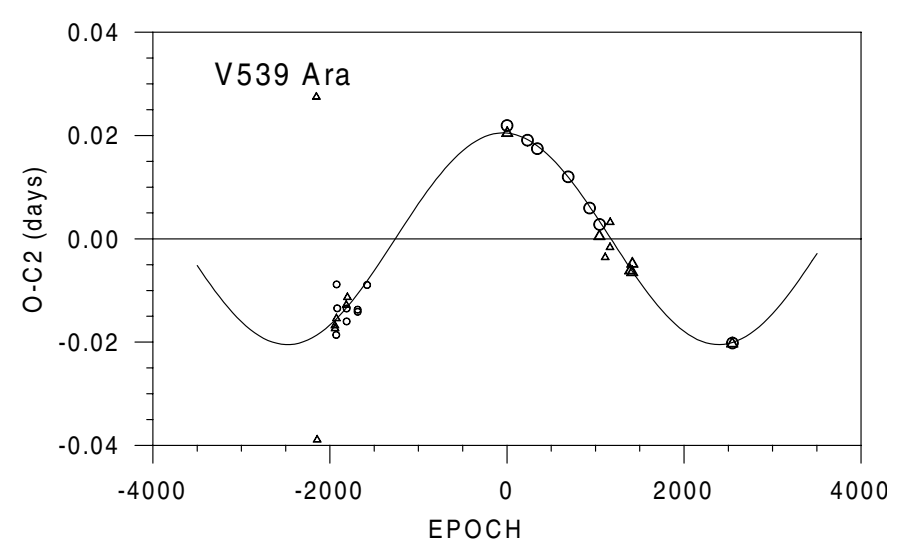

Fig. 2. The $\mathrm{O}-\mathrm{C}_{2}$ diagram for the times of minima of V539 Ara after subtraction of the apsidal motion. The sinusoidal curve represents a light-time effect for the third body circular orbit with a period of 42 years and an amplitude of about 0.02 days. The individual primary and secondary minima are denoted by circles and triangles, resp.

Subtracting the influence of apsidal motion, the $\mathrm{O}-\mathrm{C}_{2}$ diagram in Fig. 2 can be plotted. The sinusoidal variation of these values are remarkable and could be caused by a LITE. A preliminary analysis of the third body orbit gives the following parameters:

$$
\begin{array}{ll}
P_{3}(\text { period }) & =15440 \pm 310 \text { days } \\
& =42.3 \pm 0.8 \text { years } \\
T_{0}(\text { time of periastron }) & =\mathrm{JD} 2450000 \pm 180 \\
A(\text { semiamplitude }) & =0.0205 \pm 0.0012 \text { day } \\
e_{3}(\text { eccentricity }) & =0.0(\text { fixed }) \\
\omega_{3}(\text { length of periastron }) & =208.5 \pm 0.5 .
\end{array}
$$

Assuming a coplanar orbit $\left(i_{3}=90^{\circ}\right)$ and a total mass of the eclipsing pair $M_{1}+M_{2}=11.55 M_{\odot}($ Clausen 1996), we can obtain a lower limit for the mass of the third component $M_{3 \text {,min }}$. The value of the mass function is $f(M)=0.025 M_{\odot}$, from which the minimum mass of the third body follows as $1.63 M_{\odot}$. A possible third component of spectral type A8 with the bolometric magnitude of about +2.75 mag would be barely detectable in the system with a B3 primary $\left(M_{\mathrm{bol}}=-3.27 \mathrm{mag}\right.$, Harmanec 1988). For a total mass of this triple system $M_{1}+$ $M_{2}+M_{3} \simeq 13.2 M_{\odot}$ and given the value of $P_{3}$ one can obtain $a_{3}=28.7 \mathrm{AU}$. The close fainter visual companion HR 6622B $\left(m_{V}=9.3 \mathrm{mag}\right.$, sep. $\left.=12.6^{\prime \prime}\right)$ at a distance of about $3200 \mathrm{AU}$ from the eclipsing pair $(\pi=3.96 \pm 0.94$ mas, Perryman et al. 1997) clearly cannot be this third body. Therefore, new 


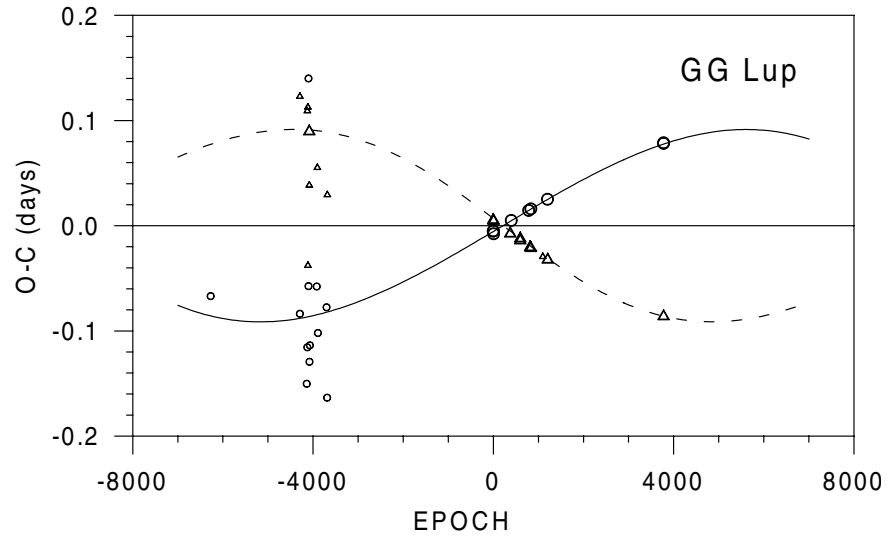

Fig. 3. The O-C diagram for the times of minimum of GG Lup. See legend to Fig. 1.

high-accuracy timings of this eclipsing binary are necessary in order to confirm the LITE in this system.

\subsection{GG Lup}

The detached eclipsing binary GG Lup (also HD 135876, HR 5687, HIP 74950, CD-40 9496, BV 448, FL 1762; $\alpha_{2000}=15^{\mathrm{h}} 58^{\mathrm{m}} 56^{\mathrm{s}}, \delta_{2000}=-40^{\circ} 47^{\prime} 18^{\prime \prime}, V_{\max }=5^{\mathrm{m}} .589 ;$ $\mathrm{Sp} . \mathrm{B} 7 \mathrm{~V}+\mathrm{B} 9 \mathrm{~V})$ is a relatively less-studied early-type binary with an eccentric orbit $(e=0.15)$ and a period of about 1.85 days. It was discovered to be a spectroscopic binary by Neubauer (1930b) and eclipses were later independently discovered by Smith (1966) and Strohmeier (1967). Photometric orbits and apsidal motion parameters were derived by Andersen et al. (1993), who found a relatively short period of apsidal motion of about $U=101 \pm 4$ years. The epochs in Table 2 were computed according to the following linear ephemeris given in that paper:

Pri. Min. $=$ HJD $2446136.7398+1.84960 \cdot$ E.

All photoelectric times of minimum light given in Andersen et al. (1993) were incorporated in our calculation. Using the Hipparcos photometry (Perryman et al. 1997), we were able to derive one additional time of minimum light. A total of 40 photoelectric times of minimum light were used in our analysis, with 18 secondary eclipses among them. The orbital inclination was adopted to be $i=78^{\circ} .0$ based on the photometric analysis (Andersen et al. 1993). The computed apsidal motion parameters and their internal errors of the least squares fit are given in Table 3. The $\mathrm{O}-\mathrm{C}$ diagram is shown in Fig. 3.

\subsection{V526 Sgr}

The detached eclipsing binary V526 Sgr (also HD 177994, $\mathrm{CD}-31^{\circ} 16374$, CPD $-31^{\circ} 5894$, FL 2634; $\alpha_{2000}=$ $19^{\mathrm{h}} 08^{\mathrm{m}} 15.0^{\mathrm{s}}, \quad \delta_{2000}=-31^{\circ} 20^{\prime} 55.0^{\prime \prime}, \quad V_{\max }=9^{\mathrm{m}} .805$; Sp. B9.5V+A2V) is a classical example of apsidal motion. It was one of the first binaries recognized to show the effects of apsidal motion in the times of its eclipses. It was discovered photographically to be a variable star in 1934 by O'Connell (1935) on plates taken at Riverview Observatory. The early

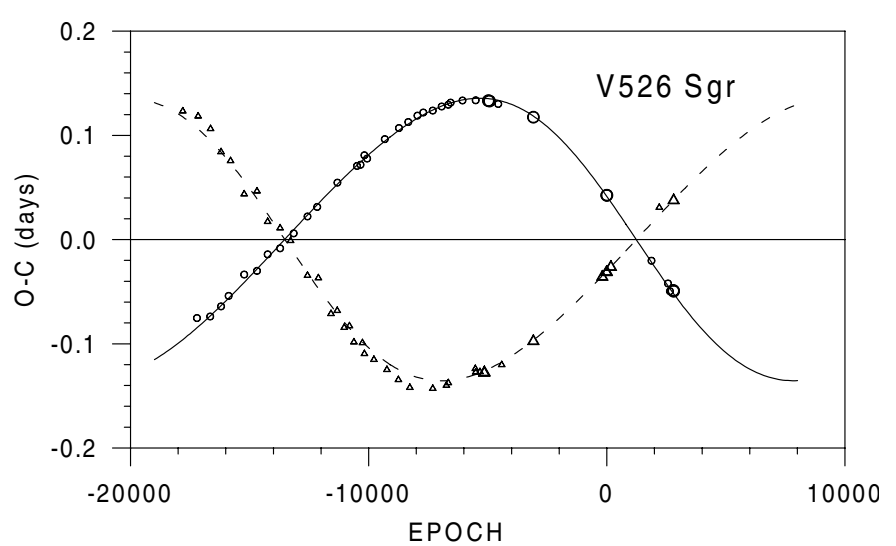

Fig. 4. The O-C diagram for the times of minima of V526 Sgr. See legend to Fig. 1.

history of this binary is described in detail in O'Connell (1967), where the following linear light elements are also given

Pri. Min. = HJD $2422054.0856+1$ 1.9194057 · E.

The improved photometric orbit and apsidal motion parameters were derived by Lacy (1993). He found a period of apsidal motion of $U=156.0 \pm 0.3$ years. Absolute dimensions and masses of V526 Sgr were later derived spectroscopicaly by Lacy (1997). The epochs in Table 2 were computed according to the ephemeris given in that paper:

Pri. Min. = HJD $2447739.69412+1$ 1.91937969 $\cdot$ E.

A total of 77 times of minimum light were used in our analysis, with 39 secondary eclipses among them. The orbital inclination was adopted to be $i=89^{\circ} .1$ based on the photometric analysis (Lacy 1993). The computed apsidal motion parameters and their internal errors of the least squares fit are given in Table 3. The $\mathrm{O}-\mathrm{C}$ diagram is shown in Fig. 4.

\section{4. $\mathrm{AO} \mathrm{Vel}$}

The detached eclipsing binary AO Vel (also HD 68826, CD $-48^{\circ} 3586$, CPD $-48^{\circ} 1553$, FL 932; $\alpha_{2000}=8^{\mathrm{h}} 11^{\mathrm{m}} 53.9^{\mathrm{s}}$, $\delta_{2000}=-48^{\circ} 44^{\prime} 46.0^{\prime \prime}, V_{\max }=9^{\mathrm{m}} .34$; Sp. B9III/Ap) is a relatively seldom-investigated early-type binary with a short period of 1.58 days in a multiple stellar system. It was discovered to be variable by Hertzsprung (1937). Apsidal motion with a short period of about 50 years was first detected by Oosterhoff \& van Houten (1949). Four-colour uvby photometry was obtained by Grønbech (1987). Clausen et al. (1995) presented the first photometric analysis and derived an apsidal motion period of $U=56.8 \pm 1.9 \mathrm{yr}$. They also discovered a third body in this system orbiting with the period of $P_{3}=25.6 \pm 2.5 \mathrm{yr}$ and contributing about $30 \%$ of the total light. As far as we know, radial velocity observations of this system are not available. AO Vel is also mentioned as one of several known Ap eclipsing binaries and the only example of the Si-type (Clausen et al. 1995).

All photoelectric times of minimum light given in Clausen et al. (1995, their Table 2) were included in our analysis. The older photographic minima (Oosterhoff \& van Houten 1949) have not been included in this apsidal motion study due to their 


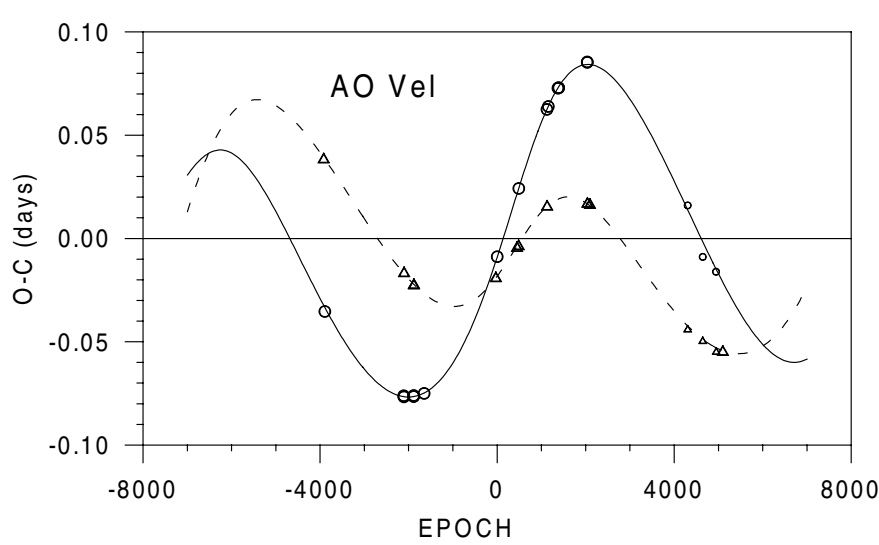

Fig. 5. The O-C diagram for the times of minimum of AO Vel. See legend to Fig. 1.

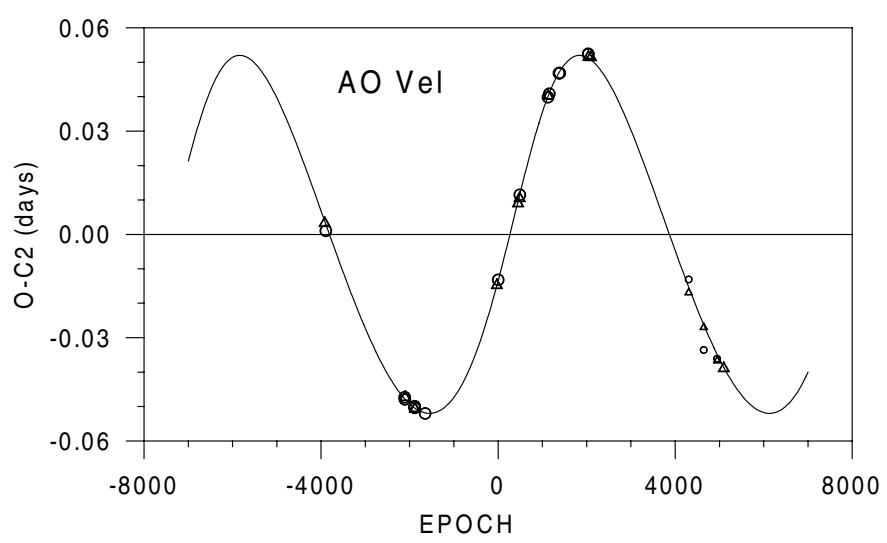

Fig. 6. The LITE for AO Vel after subtraction of the apsidal motion. The curve represents the third body orbit with a period of 33 years and an eccentricity of $e=0.2$. See legend to Fig. 2 .

large uncertainties of at least 0.05 days. The epochs in Table 2 were computed according to the ephemeris given by Clausen et al. (1995):

Pri. Min. = HJD $2445043.6806+1$ d.584660 $\cdot$ E,

Sec. Min. $=$ HJD $2445044^{\mathrm{d}} 4587+1^{\mathrm{d}} .584631 \cdot$ E.

A total of 33 photoelectric times of minimum light were used in our analysis, with 14 secondary eclipses among them. The orbital inclination was adopted to be $i=88^{\circ} .5$ based on the photometric analysis (Clausen et al. 1995). As in the case of V539 Ara we solved the apsidal motion and the LITE simultaneously. The computed apsidal motion parameters and their internal errors of the least squares fit are given in Table 3. The $\mathrm{O}-\mathrm{C}$ diagram is shown in Fig. 5. An analysis of the third body orbit gives the following parameters:
$P_{3}$ (period)
$=12170 \pm 200$ days
$=33.3 \pm 0.5$ years
$T_{0}$ (time of periastron)
$=\mathrm{JD} 2446225 \pm 200$
$A$ (semiamplitude)
$=0.052 \pm 0.004$ day
$=0.207 \pm 0.012$
$\omega_{3}$ (length of periastron) $=27.5 \pm 0.3$.

Assuming a coplanar orbit $\left(i_{3}=90^{\circ}\right)$ and a total mass of the eclipsing pair $M_{1}+M_{2}=8.0 M_{\odot}$ (Clausen et al. 1995), we obtained the value of the mass function of $f(M)=0.692 M_{\odot}$, from which the minimum mass of the third body is $M_{3, \min }=$ $4.40 M_{\odot}$. The third component of spectral type B5 with bolometric magnitude of about -2.7 mag can produce more than $30 \%$ of the total light in the system with a B9III primary $\left(M_{\mathrm{bol}}=-1.3 \mathrm{mag}\right.$, Schmidt-Kaler 1982).

\section{Internal structure constant}

The internal structure constant (ISC) $k_{2}$, which is related to the variation of the density within the star, is an important parameter of stellar evolution models. However, the period of rotation of the periastron in eccentric eclipsing binaries does not allow us to derive the individual internal stellar constant of the component stars. The observed average value of $k_{2, \text { obs }}$ can be derived using the following expression:

$k_{2, \mathrm{obs}}=\frac{1}{c_{21}+c_{22}} \frac{P_{a}}{U}=\frac{1}{c_{21}+c_{22}} \frac{\dot{\omega}}{360}$,

where $c_{21}$ and $c_{22}$ are functions of the orbital eccentricity, fractional radii, the masses of the components and the ratio between rotational velocity of the stars and Keplerian velocity (Kopal 1978). We also assume that the component stars rotate pseudosynchronously with the same angular velocity as the maximum orbital value at periastron. Taking into account the value of the eccentricity and the masses of the components, one has to subtract from $\dot{\omega}$ a relativistic correction $\dot{\omega}_{\text {rel }}$ (Levi-Civita 1937; Giménez 1985):

$\dot{\omega}_{\text {rel }}=5.45 \times 10^{-4} \frac{1}{1-e^{2}}\left(\frac{M_{1}+M_{2}}{P}\right)^{2 / 3}$,

where $M_{i}$ denotes the individual masses of the components in solar units and $P$ is the orbital period in days. The values of $\dot{\omega}_{\text {rel }}$ and the resulting mean internal structure constants $k_{2, \text { obs }}$ are given in Table 4 . Their errors were evaluated according to the following relation

$\Delta \log k_{2, \mathrm{obs}} \simeq 0.434\left(\frac{\Delta \dot{\omega}}{\dot{\omega}}+5 \frac{\frac{\Delta r_{1}}{r_{1}} c_{21}+\frac{\Delta r_{2}}{r_{2}} c_{22}}{c_{21}+c_{22}}\right)$,

where $\Delta \dot{\omega}$ and $\Delta r_{1,2}$ are standard errors of the apsidal motion rate and fractional radii of binary components. The uncertainities in the mass and in the eccentricity were neglected due to the presence of higher terms in the eccentricity. The complete formula for the calculation of the ISC error is given in the paper of Tudose at al. (2000).

The presence of a third component with orbital period $P_{3}$ perturbs the orbit of a close binary system. One of the most sensitive elements is the longitude of the periastron. The acceleration of the rate of apsidal motion for coplanar orbits and small eccentricities caused by the presence of the third body $\dot{\omega}_{3}$ can be approximated by (Martynov 1948):

$\dot{\omega}_{3}=\frac{3}{8} \lambda m^{2}+\frac{225}{32} \lambda^{2} m^{3}$,

where

$\lambda=\frac{M_{3}}{M_{1}+M_{2}+M_{3}}, \quad$ and $\quad m=\frac{P_{s}}{P_{3}}$. 
Table 4. Adopted basic physical properties of the binary components and the internal structure constant.

\begin{tabular}{lccccc}
\hline \hline Parameter & Unit & V539 Ara & GG Lup & V526 Sgr & AO Vel \\
\hline$M_{1}$ & $M_{\odot}$ & $6.24(7)$ & $4.119(40)$ & $2.27(7)$ & 4.4 \\
$M_{2}$ & $M_{\odot}$ & $5.31(6)$ & $2.509(24)$ & $1.68(6)$ & 3.6 \\
$r_{1}$ & & $0.220(4)$ & $0.2000(20)$ & $0.1843(4)$ & $0.214(6)$ \\
$r_{2}$ & & $0.167(4)$ & $0.1450(15)$ & $0.1521(8)$ & $0.193(6)$ \\
$i$ & deg & $85.2(2)$ & $86.75(10)$ & $89.1(3)$ & $85.5(1.5)$ \\
\hline Source & & Clausen & Andersen & Lacy & Clausen \\
& & $(1996)$ & et al. $(1993)$ & $(1997)$ & et al. $(1995)$ \\
\hline$\dot{\omega}_{\text {rel }}$ & deg cycle ${ }^{-1}$ & 0.00129 & 0.00131 & 0.00093 & 0.00161 \\
$\dot{\omega}_{\text {rel }} / \dot{\omega}$ & $\%$ & 6.7 & 7.3 & 7.6 & 5.8 \\
$\log k_{2, \text { obs }}$ & & -2.326 & -2.157 & -2.357 & -2.243 \\
$\Delta \log k_{2, \text { obs }}$ & & 0.065 & 0.034 & 0.012 & 0.069 \\
$\log k_{2, \text { theo }}$ & & -2.30 & -2.18 & -2.41 & -2.15 \\
$Z$ (metal abundance) & & 0.02 & 0.015 & 0.02 & 0.02 \\
\hline
\end{tabular}

This correction for the apsidal motion is negligible in both systems (V539 Ara and AO Vel) due to the relatively long period $P_{3}$ of the third body orbit.

Theoretical values $k_{2, \text { theo }}$ according to available theoretical models for the internal stellar structure computed by Claret $\&$ Giménez (1992) for given masses of components are presented in Table 4. The metal abundance $Z$ for $X=0.7$ is also given.

\section{Conclusions}

The photometric study of triple or multiple stellar systems with apsidal motion of the eclipsing pair is a relatively poorly studied field of celestial mechanics. This study provides accurate information on the apsidal motion rates and internal structure constants of the three main-sequence binary systems V539 Ara, GG Lup and V526 Sgr and possible B9III/Ap system AO Vel. None of the analysed binaries present an important relativistic contribution. The obtained values of the internal structure constant $k_{2 \text {,obs }}$ are compared to their theoretical values $k_{2 \text {,theo }}$ according to available theoretical models for the internal stellar structure along the main sequence computed by Claret $\&$ Giménez (1992) for a variety of masses and chemical compositions (Fig. 7).

The apsidal motion in eccentric eclipsing binaries has been used for decades to test evolutionary stellar models. Extensive comparison between theoretical models of stellar structure, including moderate core overshooting and mass loss, and the best available data of apsidal motion rates for double-lined eclipsing binaries was done by Claret \& Giménez (1993), using the opacities published by Rogers \& Iglesias (1992). They concluded that there were no systematic deviations between observational and theoretical values of $\log k_{2}$. The previous discrepancies between theory and observations have been reduced to an acceptable level with the introduction of modern stellar models including the effects of dynamic tides (Claret \& Willems 2002). Recently, new grids of stellar models based on updated opacities, expanded nuclear network and mass loss rates were published by Claret (2004).

With the exception of the system AO Vel, where the stellar masses are probably estimated incorrectly, the agreement

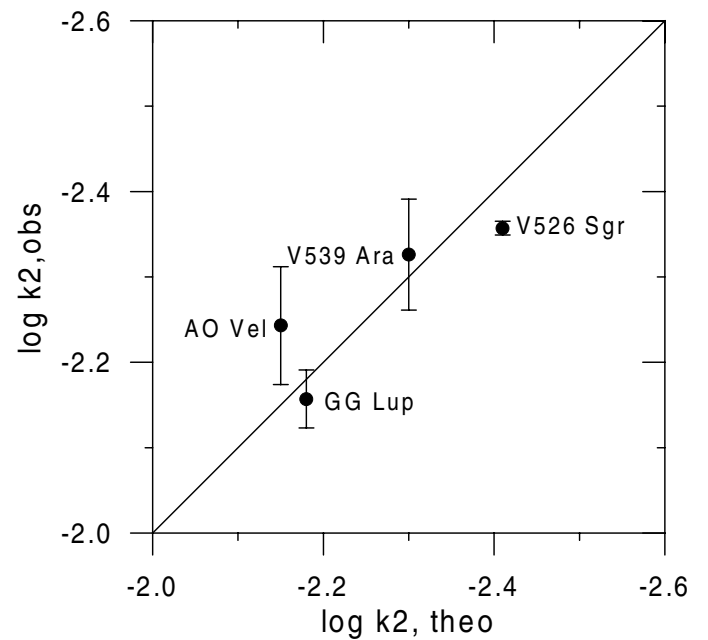

Fig. 7. Comparison between observed and predicted average values of $\log k_{2}$ using models of stellar evolution.

between the theoretical and observed value of the ISC is very good for V539 Ara and GG Lup and satisfactory for V526 Sgr.

For V539 Ara we derived a slightly longer apsidal motion period of about $U=162 \pm 8$ years then was given in Clausen (1996). For GG Lup we have confirmed the apsidal motion period of $U=102 \pm 1$ years, which is in excellent agreement with the results obtained by Andersen et al. (1993). Our results for the apsidal motion of V526 Sgr are practical identical to the parameters derived in Lacy (1993).

For AO Vel we substantially improved the apsidal motion and LITE parameters. The third component which has a wide orbit ( $a \sin i=9.2 \mathrm{AU}$ ) probably contributes more than $30 \%$ of the total light of this multiple system. It is also highly desirable to obtain new, high-dispersion and high-S/N spectroscopic observations for this system and to apply modern disentangling methods to them to obtain the radial-velocity curves of both binary components and, therefore, derive accurate masses for this important system. This massive system with its relatively short orbital period could be attractive for spectroscopists. The radial velocity curve should have a semi-amplitude of more than $200 \mathrm{~km} \mathrm{~s}^{-1}$. 
Moreover, V539 Ara and AO Vel belong to the important group of triple eccentric eclipsing binaries with apsidal motion (f.e. V889 Aql, TV Cet, RU Mon, U Oph, YY Sgr, DR Vul) described recently in Wolf et al. (2001). New high-accuracy timings of these eclipsing binaries are necessary to improve the LITE parameters derived in this paper.

Acknowledgements. This investigation was supported by the Grant Agency of the Czech Republic, grant No. 205/04/2063 and by allocation of SAAO observing time. The research of MW was also supported by the plan J13/98: 113200004 of Ministry of Education, Youth and Sports. MW wish to thank the staff at SAAO for their warm hospitality and help with the equipment and Dr. Petr Harmanec for help with the photometric reductions. We are indebted to the referee Dr. J. V. Clausen for his helpful comments that improved the paper. This research has made use of the SIMBAD database, operated at CDS, Strasbourg, France, and of NASA's Astrophysics Data System Bibliographic Services.

\section{References}

Andersen, J., Clausen, J. V., \& Giménez, A. 1993, A\&A, 277, 439

Claret, A., \& Giménez, A. 1992, A\&AS, 96, 255

Claret, A., \& Giménez, A. 1993, A\&A, 277, 487

Claret, A., \& Willems, B. 2002, A\&A, 388, 518

Claret, A. 2004, A\&A, 424, 919

Clausen, J. V. 1991, A\&A, 246, 397

Clausen, J. V. 1996, A\&A, 308, 151

Clausen, J. V., Giménez A., \& van Houten, C. J. 1995, A\&AS, 109, 425

Cowling, T. G. 1938, MNRAS, 98, 734

Giménez, A. 1985, ApJ, 297, 405

Giménez, A., \& Bastero, M. 1995, Ap\&SS, 226, 99

Giménez, A., \& García-Pelayo, J. M. 1983, Ap\&SS, 92, 203

Grønbech B. 1987, A\&AS, 68, 317

Harmanec, P. 1988, Bull. Astr. Inst. Czech., 39, 329
Harmanec, P., \& Horn, J. 1998, Journal of Astronomical Data, 4, CD-ROM file 5, ed. C. Sterken, Vrije Universiteit Brussel

Hertzsprung, E. 1937, BAN, 8, 157

Kopal, Z. 1978, Dynamics of Close Binary Systems (Dordrecht, Holland: Reidel)

Kreiner, J. M., Kim, C.-H., \& Nha, I.-S. 2001, An Atlas of O-C diagrams of Eclipsing Binary Stars (Cracow, Poland: Wydawnictwo Naukowe Akademii Pedagogicznej)

Lacy, C. H. S. 1993, AJ, 105, 1096

Lacy, C. H. S. 1997, AJ, 113, 1091

Levi-Civita, T. 1937, Amer. J. Math., 59, 225

Martynov, D. Ya. 1948, Izv. Engelhardt Obs. Kazan, No. 25

Menzies, J. W., Cousins, A. W., Banfield, R. M., \& Laing, J. D. 1989, SAAO Circ., 13, 1

Neubauer, F. J. 1930a, Lick Obs. Bull., 15, 46

Neubauer, F. J. 1930b, PASP, 42, 235

O'Connell, D. J. K. 1935, Astr. Nachr., 257, 391

O’Connell, D. J. K. 1967, Ricerche Astron., 7, 339

Oosterhoff, P. Th., \& van Houten, C. J. 1949, BAN, 11, 63

Perryman, M. A. C, Høg, E., Kovalevsky, J., Lindegren, L., \& Turon, C. 1997, The Hipparcos and Tycho Catalogues, ESA SP-1200

Pojmanski, G. 2002, Acta Astron., 52, 397

Rogers, R. J., \& Iglesias, C. A. 1992, ApJS, 79, 507

Schmidt-Kaler, T. H. 1982, in Landolt-Bornstein New Series, Vol 2b, Stars and Star Cluster, ed. K. Schaifers, \& H. H. Voigt (Springer)

Smith, L. F. 1966, PASP, 78, 168

Sterne, T. E. 1939, MNRAS, 99, 662

Strohmeier, W. 1964, IBVS, No. 49

Strohmeier, W. 1967, IBVS, No. 208

Tudose, V., Dumitrescu, A., \& Rusu, M. 2000, Rom. Astron. J., 10, 35

Wolf, M. 2000, A\&A, 356, 134

Wolf, M., Diethelm, R., \& Hornoch, K. 2001, A\&A, 374, 243

Wolf, M., Diethelm, R., \& Šarounová, L. 1999, A\&A, 345, 553

Wolf, M., \& Šarounová, L. 1995, A\&AS, 114, 143

Wozniak, P. R., Vestrand, W. T., Akerlof, C. W., et al. 2004, AJ, 127, 2436 